ISSN 1855-3966 (printed edn.), ISSN 1855-3974 (electronic edn.)

ARS MATHEMATICA CONTEMPORANEA 15 (2018) 19-37

https://doi.org/10.26493/1855-3974.1376.7c2

(Also available at http://amc-journal.eu)

\title{
On Wiener inverse interval problem of trees
}

\author{
Jelena Sedlar* \\ University of Split, Faculty of civil engineering, architecture and geodesy, \\ Matice hrvatske 15, 21000 Split, Croatia
}

Received 6 April 2017, accepted 30 September 2017, published online 2 November 2017

\begin{abstract}
The Wiener index $W(G)$ of a simple connected graph $G$ is defined as the sum of distances over all pairs of vertices in a graph. We denote by $W\left[\mathcal{T}_{n}\right]$ the set of all values of the Wiener index for a graph from the class $\mathcal{T}_{n}$ of trees on $n$ vertices. The largest interval of consecutive integers (consecutive even integers in case of odd $n$ ) contained in $W\left[\mathcal{T}_{n}\right]$ is denoted by $W^{i n t}\left[\mathcal{T}_{n}\right]$. In this paper we prove that both sets are of cardinality $\frac{1}{6} n^{3}+O\left(n^{5 / 2}\right)$ in the case of even $n$, while in the case of odd $n$ we prove that the cardinality of both sets equals $\frac{1}{12} n^{3}+O\left(n^{5 / 2}\right)$, which essentially solves two conjectures posed in the literature.
\end{abstract}

Keywords: Wiener index, Wiener inverse interval problem, Tree.

Math. Subj. Class.: 05C05, 05C90

\section{Introduction}

The Wiener index of a connected graph $G$ is defined as the sum of distances over all pairs of vertices, i.e.

$$
W(G)=\sum_{u, v \in V(G)} d(u, v) .
$$

It was first introduced in [13] and it was used for predicting the boiling points of paraffins. Since the index was very successful many other topological indices were introduced later which use the distance matrix of a graph. There is a recent survey by Gutman et al. [14] in which finding extremal values and extremal graphs for the Wiener index and several of its variations is nicely presented. Given the class of all simple connected graphs on $n$ vertices it is easy to establish extremal graphs for the Wiener index, those are complete graph $K_{n}$ and path $P_{n}$. The same holds for the class of tree graphs on $n$ vertices in which the minimal

\footnotetext{
* This work has been supported in part by Croatian Science Foundation under the project 8481 (BioAmpMode) and Croatian-Chinese bilateral project "Graph-theoretical methods for nanostructures and nanomaterials". Also, many thanks to the anonymous reviewers for careful reading of the paper and very valuable suggestions.

E-mail address: jsedlar@gradst.hr (Jelena Sedlar)
} 
tree is the star $S_{n}$ and the maximal tree is the path $P_{n}$. Many other bounds on the Wiener index are also established in the literature.

In [4] Gutman and Yeh proposed the inverse Wiener index problem, i.e. for a given value $w$ the problem of finding a graph (or a tree) $G$ for which $W(G)=w$. The first attempt at solving the problem was made in [7] where integers up to 1206 were checked and 49 integers were found that are not Wiener indices of trees. In [1] it was computationally proved that for all integers $w$ between $10^{3}$ and $10^{8}$ there exists a tree with Wiener index $w$. The problem was finally fully solved in 2006 when two papers were published solving the problem independently. It was proved in [12] that for every integer $w>108$ there is a caterpillar tree $G$ such that $W(G)=w$. The other proof is from the paper [9] where it was proved that all integers except those 49 are Wiener indices of trees with diameter at most 4 . Since the most interesting graphs to be considered are chemical trees (especially those in which maximum vertex degree is at most 3) and hexagon type graphs, in [11] this problem was further considered on classes of such graphs.

A related question is to ask what value of the Wiener index can a graph (or a tree) $G$ on $n$ vertices have? In order to clarify further this problem one may also ask how many such values are there, how are they distributed along the related interval or how many of them are consecutive. In [6] this problem is named the Wiener inverse interval problem (see also a nice recent survey [5] which covers the topic). In that paper the set $W\left[\mathcal{G}_{n}\right]$ is defined as the set of all values of the Wiener index for graphs $G \in \mathcal{G}_{n}$, where $\mathcal{G}_{n}$ is the class of simple connected graphs on $n$ vertices. Similarly, $W\left[\mathcal{T}_{n}\right]$ is defined as the set of values $W(T)$ for all trees on $n$ vertices $\left(\mathcal{T}_{n}\right.$ denotes the class of trees on $n$ vertices). Also, $W^{\text {int }}\left[\mathcal{G}_{n}\right]$ (or analogously $\left.W^{i n t}\left[\mathcal{T}_{n}\right]\right)$ is defined to be the largest interval of consecutive integers such that $W^{\text {int }}\left[\mathcal{G}_{n}\right] \subseteq W\left[\mathcal{G}_{n}\right]$ (or analogously $W^{\text {int }}\left[\mathcal{T}_{n}\right] \subseteq W\left[\mathcal{T}_{n}\right]$ ).

In [6] the Wiener inverse interval problem on the class $\mathcal{G}_{n}$ was considered. First, the authors noted that obviously $W^{i n t}\left[\mathcal{G}_{n}\right] \subseteq W\left[\mathcal{G}_{n}\right] \subseteq\left[W\left(K_{n}\right), W\left(P_{n}\right)\right]$. Since $W\left(K_{n}\right)$ and $W\left(P_{n}\right)$ are easily computed, the upper bound $\left|W^{i n t}\left[\mathcal{G}_{n}\right]\right| \leq\left|W\left[\mathcal{G}_{n}\right]\right| \leq \frac{n^{3}}{6}-\frac{n^{2}}{2}+\frac{n}{3}+1$ easily follows. Introducing dandelion and comet graphs and establishing how the values between the values of the Wiener index for dandelion and comet graph can be obtained, the authors obtain the following lower bound $\left|W\left[\mathcal{G}_{n}\right]\right| \geq\left|W^{i n t}\left[\mathcal{G}_{n}\right]\right| \geq \frac{n^{3}}{6}-\frac{5}{2} n^{2}-\frac{1}{3} n^{3 / 2}+$ $\frac{19}{3} n+\frac{7}{3} n^{1 / 2}$. These bounds sandwich the value of $\left|W^{\text {int }}\left[\mathcal{G}_{n}\right]\right|$ and $\left|W\left[\mathcal{G}_{n}\right]\right|$ in terms of $n^{3}$ tightly, therefore the result $\left|W^{\text {int }}\left[\mathcal{G}_{n}\right]\right|=\left|W^{i n t}\left[\mathcal{G}_{n}\right]\right|=\frac{n^{3}}{6}+O\left(n^{2}\right)$ easily follows. The authors further conjecture that $\left|W\left[\mathcal{G}_{n}\right]\right|=\frac{n^{3}}{6}-\frac{n^{2}}{2}+\Theta(n)$. Regarding the same problem on the class $\mathcal{T}_{n}$ the following two conjectures were made.

Conjecture 1.1. The cardinality of $W\left[\mathcal{T}_{n}\right]$ equals $\frac{1}{6} n^{3}+\Theta\left(n^{2}\right)$.

Conjecture 1.2. The cardinality of $W^{\text {int }}\left[\mathcal{T}_{n}\right]$ equals $\Theta\left(n^{3}\right)$.

In this paper we will consider these two conjectures. First, we will note that for a tree $T$ on odd number of vertices $n$ the value $W(T)$ can be only an even number. That means that the Wiener inverse interval problem in that case has to be reformulated as the problem of finding the largest interval $W^{\text {int }}\left[\mathcal{T}_{n}\right]$ of consecutive even integers such that $W^{i n t}\left[\mathcal{T}_{n}\right] \subseteq W\left[\mathcal{T}_{n}\right]$. Since $\left|W\left[\mathcal{T}_{n}\right]\right| \leq W\left(P_{n}\right)-W\left(S_{n}\right)+1=\frac{1}{6} n^{3}-n^{2}+\frac{11}{6} n$, we now conclude that the cardinality of $W\left[\mathcal{T}_{n}\right]$ in the case of odd $n$ can be at most $\frac{1}{12} n^{3}+O\left(n^{2}\right)$. Given that reformulation, we will prove both conjectures to be true in terms of $n^{3}$. Even more, we will prove the strongest possible version of Conjecture 1.2 in terms of $n^{3}$ by proving that $\left|W^{i n t}\left[\mathcal{T}_{n}\right]\right|$ also equals $\frac{1}{6} n^{3}+O\left(n^{5 / 2}\right)$ (i.e. $\frac{1}{12} n^{3}+O\left(n^{5 / 2}\right)$ in case of odd 
$n$ ) which is the best possible result given the upper bound on $\left|W\left[\mathcal{T}_{n}\right]\right|$ derived from the difference between $W\left(P_{n}\right)$ and $W\left(S_{n}\right)$. These results will yield quite a strong result for the class $\mathcal{T}_{n}^{4}$ of chemical trees as a direct corollary.

The present paper is organized as follows. In the next section basic definitions and preliminary results are given. In the third section the problem is solved for trees on even number of vertices, while in the fourth section the problem is solved for trees on odd number of vertices. In the fifth section we conclude the paper with several remarks and possible directions for further research.

\section{Preliminaries}

Let $G=(V(G), E(G))$ be a simple connected graph having $n=|V(G)|$ vertices and $m=|E(G)|$ edges. For a pair of vertices $u, v \in V(G)$ we define the distance $d_{G}(u, v)$ as the length of the shortest path connecting $u$ and $v$ in $G$. For a vertex $u \in V(G)$ the degree $d_{G}(u)$ is defined as the number of neighbors of vertex $u$ in graph $G$. When it doesn't lead to confusion we will use the abbreviated notation $d(u, v)$ and $d(u)$. Also, for a vertex $u \in$ $V(G)$ and a set of vertices $A \subseteq V(G)$ we will denote $d(u, A)=\sum_{v \in A} d(u, v)$. Similarly, for two sets of vertices $A, B \subseteq V(G)$ we will denote $d(A, B)=\sum_{u \in A, v \in B} d(u, v)$. We say that a vertex $u \in V(G)$ is a leaf if $d_{G}(u)=1$, otherwise we will say that $u$ is an interior vertex of a graph $G$. A graph $G$ which does not contain cycles is called a tree. A tree graph will usually be denoted by $T$ throughout the rest of the paper. We say that a tree $T$ is a caterpillar tree if all its interior vertices induce a path. Such a path will be called the interior path of a caterpillar. Let $a$ and $b$ be positive integers such that $a \leq b$. We say that the interval $[a, b]$ is Wiener $p$-complete if there is a tree $T$ in $\mathcal{T}_{n}$ such that $W(T)=a+p i$ for every $i=0, \ldots,\left\lfloor\frac{b-a}{p}\right\rfloor$. We say that the interval $[a, b]$ is Wiener complete if it is Wiener 1 -complete.

Let us now note that the value of the Wiener index for a tree $T$ on odd number of vertices $n$ is an even number. There are various ways to prove this fact, maybe the simplest one is to recall that for a tree $T$ on $n$ vertices it holds that

$$
W(T)=\sum_{u v \in E(T)} n_{u} \cdot n_{v}
$$

where $n_{u}$ and $n_{v}$ are the number of vertices in the connected component of $T \backslash\{u v\}$ containing $u$ and $v$ respectively. Obviously, $n_{u}+n_{v}=n$ and therefore in the case of odd $n$ the product $n_{u} \cdot n_{v}$ must be an even number. I would like here to thank prof. Tomislav Došlić for suggesting this short proof to me and to the anonymous reviewers for referring me to the interesting survey [2] in which this fact is already explained and to several interesting papers ([3], [8] and [10]) in which one can read more on the subject. Before proceeding further, let us state this fact as a formal theorem which we can reference in further text.

Theorem 2.1. Let $T$ be a tree on odd number of vertices $n \geq 3$. Then $W(T)$ is an even number.

The main tool for obtaining our results throughout the paper will be a transformation of a tree which increases the value of the Wiener index by exactly four. We will call it Transformation A, but let us introduce its formal definition.

Definition 2.2. Let $T$ be a tree and $u \in V(T)$ a vertex of degree 4 such that neighbors $v_{1}$ and $v_{2}$ of $u$ are leaves, while neighbors $w_{1}$ and $w_{2}$ of $u$ are not leaves. We say that a tree 
$T^{\prime}$ is obtained from $T$ by Transformation $\mathrm{A}$ if $T^{\prime}$ is obtained from $T$ by deleting edges $u v_{1}$ and $u v_{2}$, while adding edges $w_{1} v_{1}$ and $w_{2} v_{2}$.

Theorem 2.3. Let $T$ be a tree and let $T^{\prime}$ be a tree obtained from $T$ by Transformation A. Then $W\left(T^{\prime}\right)=W(T)+4$.

Proof. For simplicity's sake we will use the notation $d^{\prime}(u, v)$ for $d_{T^{\prime}}(u, v)$. Let $T_{w_{i}}=$ $\left(V_{w_{i}}, E_{w_{i}}\right)$ be the connected component of $T \backslash\{u\}$ which contains vertex $w_{i}$ for $i=1,2$. Note that the only distances that change in Transformation A are distances from vertices $v_{1}$ and $v_{2}$. For every $v \in V_{w_{1}} \cup V_{w_{2}}$ we have

$$
d^{\prime}\left(v_{1}, v\right)-d\left(v_{1}, v\right)+d^{\prime}\left(v_{2}, v\right)-d\left(v_{2}, v\right)=0 .
$$

For the vertex $u$ we have

$$
d^{\prime}\left(v_{1}, u\right)-d\left(v_{1}, u\right)+d^{\prime}\left(v_{2}, u\right)-d\left(v_{2}, u\right)=2 .
$$

Finally, we also have $d^{\prime}\left(v_{1}, v_{2}\right)-d\left(v_{1}, v_{2}\right)=2$. Therefore, $W\left(T^{\prime}\right)-W(T)=4$ which proves the theorem.

Although Transformation A can be applied on any tree graph, we will mainly apply it on caterpillar trees. Moreover, it is critical to find a kind of caterpillar tree on which Transformation A can be applied repeatedly as many times as possible. For that purpose, let us prove the following theorem.

Theorem 2.4. Let $T$ be a caterpillar tree and $P=u_{1} \ldots u_{d}$ its interior path. If there is a vertex $u_{i} \in P$ of degree 4 such that $u_{i \pm j}$ is of degree 3 for every $1 \leq j \leq k-1$, then the interval $\left[W(T), W(T)+4 k^{2}\right]$ is Wiener 4-complete.

Proof. Let us denote a caterpillar tree $T$ from the statement of the lemma by $T^{k}$ (since $1 \leq$ $j \leq k-1)$. Also, let us denote $D=\left\{u_{i \pm j}: j=0, \ldots, k-1\right\}$. To obtain the desired result we will systematically apply Transformation A to vertices from $D$ until there is no more vertices in $D$ to which Transformation A can be applied. Let us now explain into greater detail by what system that is done. First, note that in $T^{k}$ Transformation A can initially be applied only to $u_{i}$. By applying transformation A to $u_{i}$ in $T^{k}$ we will obtain a caterpillar tree in which Transformation $A$ can be applied to vertices $u_{i-1}$ and $u_{i+1}$. By applying Transformation A to $u_{i-1}$ and $u_{i+1}$ consecutively we will obtain a caterpillar tree in which Transformation A can be applied to $u_{i-2}$ and $u_{i+2}$ (and $u_{i}$ but we will not further apply Transformation A to that vertex for the time being). By further applying transformation A to $u_{i-2}$ and $u_{i+2}$ consecutively and repeating this procedure we will reach a caterpillar tree in which Transformation A can be applied to vertices $u_{i-(k-1)}$ and $u_{i+(k-1)}$ and finally apply Transformation A to those two vertices. The caterpillar tree obtained after that last step we can denote by $T^{k-1}$ because of the following: in that tree vertex $u_{i} \in P$ is of degree 4 and vertices $u_{i \pm j}$ are of degree 3 for every $j=1, \ldots, k-2$. Note that in the process of transforming $T^{k}$ to $T^{k-1}$ we will have applied the Transformation A $2 k-1$ times. Now, the same process can be repeated on $T^{k-1}$ to obtain $T^{k-2}$. The procedure stops when we reach $T^{1}$ in which $u_{i}$ is the only vertex in $D$ having degree greater than 2 (to be more precise, the degree of $u_{i}$ in $T^{1}$ equals 4 , so Transformation A can be applied to it one more time). Applying Transformation A on $u_{i}$ in $T^{1}$ we finally obtain $T^{0}$ in which Transformation A cannot be further applied to vertices from $D$. Therefore, in transforming $T^{k}$ to $T^{0}$ Transformation A was used $\sum_{j=1}^{k}(2 j-1)=k^{2}$ times and each time the value of the Wiener index increased by 4 . 
Note that the Transformation A in Theorem 2.4 is applied $k^{2}$ times on a caterpillar in which interior path is of length $d-1$. If we prove that there are $\Theta(n)$ different values of $d$ for which $k=\Theta(n)$, we obtain roughly $\Theta\left(n^{3}\right)$ graphs with different values of the Wiener index which is exactly the result we aim at (of course, here one has to be careful to avoid significant overlapping of the values of the Wiener index for caterpillars with different values of $d$ ). So, that is what we are going to do in following sections, but in order to do that with sufficient mathematical precision we will have to construct four different special types of caterpillar trees. To easily construct those four types of caterpillar trees we first introduce two basic types of caterpillars from which those four types will be constructed by adding one or two vertices.

Definition 2.5. Let $n, d$ and $x$ be positive integers such that $n \geq 18$ is even, $\left\lceil\frac{n-2}{4}\right\rceil \leq d \leq$ $\frac{n-8}{2}$ and $x \leq \frac{4+4 d-n}{2}$. Caterpillar $B_{1}(n, d, x)$ is a caterpillar on even number of vertices $n$ obtained from path $P=u_{-d} \ldots u_{-1} u_{0} u_{1} \ldots u_{d}$ by appending a leaf to vertices $u_{-d-1+x}$ and $u_{d+1-x}$ and by appending a leaf to $2 k-1$ consecutive vertices $u_{-(k-1)}, \ldots, u_{k-1}$ where $k=\frac{n-(2 d+1)-1}{2}$.

Caterpillar graph $B_{1}(n, d, x)$ is illustrated by Figure 1 (vertex $u_{i}$ of the interior path is in the images denoted just by $i$ in order to make labels easier to see).

a)

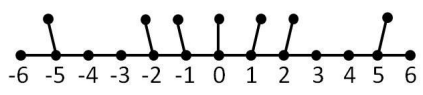

b)

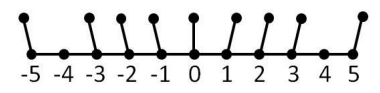

Figure 1: Caterpillar graphs: a) $B_{1}(20,6,2)$, b) $B_{1}(20,5,1)$.

Lemma 2.6. Let $n, d$ and $x$ be integers such that $B_{1}(n, d, x)$ is defined. Then

$$
\begin{aligned}
W\left(B_{1}(n, d, x)\right) & =\frac{n^{3}}{4}+\left(-\frac{3 d}{2}-\frac{5}{4}\right) n^{2}+\left(4 d^{2}+10 d+\frac{13}{2}-2 x\right) n+ \\
& +2 x^{2}-\frac{8}{3} d^{3}-12 d^{2}-\frac{46 d}{3}-7 .
\end{aligned}
$$

Proof. Let $k=\frac{n-(2 d+1)-1}{2}$ and $x^{\prime}=-d-1+x$. Even though the structure of $B_{1}$ is a bit complicated it is still regular enough so that the Wiener index can be computed exactly (as a function in variables $n, d$ and $x$ ). Let us divide vertices of $B_{1}$ into three sets $A, B$ and $C$ so that set $A$ contains vertices $u_{i}$ for $i=-d, \ldots, d$, set $B$ contains leaves attached to $2 k-1$ consecutive vertices $u_{-(k-1)}, \ldots, u_{k-1}$ and set $C$ contains two leaves attached to 
vertices $u_{-d-1+x}$ and $u_{d+1-x}$. Note that we have

$$
\begin{aligned}
& d(A, A)=\sum_{i=-d}^{d} \sum_{j=i+1}^{d}(j-i), d(B, B)=\sum_{i=-(k-1)}^{k-1} \sum_{j=i+1}^{k-1}(j-i+2) \\
& d(C, C)=(2 d+2-2(x-1)), d(A, B)=\sum_{i=-d}^{d} \sum_{j=-(k-1)}^{k-1}(|i-j|+1) \\
& d(A, C)=2 \sum_{i=-d}^{d}\left(\left|i-x^{\prime}\right|+1\right), d(B, C)=2 \sum_{i=-(k-1)}^{k-1}\left(\left|i-x^{\prime}\right|+2\right)
\end{aligned}
$$

Noting that

$$
W\left(B_{1}(n, d, x)\right)=d(A, A)+d(B, B)+d(C, C)+d(A, B)+d(A, C)+d(B, C)
$$

and simplifying the obtained sum yields the formula from the statement of the lemma.

Note that the caterpillar $B_{1}(n, d, x)$ is a caterpillar with relatively long interior path. Namely, the value $d$ is roughly half of the length of the interior path and in the definition of $B_{1}(n, d, x)$ the value of $d$ is relatively large with respect to number of vertices $n$. We now introduce the formal definition of the second basic caterpillar which will have relatively short interior path.

Definition 2.7. Let $n, d$ and $x$ be positive integers such that $n \geq 18$ is even, $4 \leq d \leq\left\lfloor\frac{n}{4}\right\rfloor$ and $x \leq \frac{n-4 d+2}{2}$. Caterpillar $B_{2}(n, d, x)$ is a caterpillar on even number of vertices $n$ obtained from path $P=u_{-d} \ldots u_{-1} u_{0} u_{1} \ldots u_{d}$ by appending a leaf to $2 k-1$ consecutive vertices $u_{-(k-1)}, \ldots, u_{k-1}$ where $k=d-1$, by appending $x$ leaves to each of the $u_{-(d-1)}$ and $u_{(d-1)}$, and by appending $r$ leaves to each of the $u_{-d}$ and $u_{d}$ where $r=\frac{n-4 d-2 x+2}{2}$.

Caterpillar graph $B_{2}(n, d, x)$ is illustrated by Figure 2 (vertex $u_{i}$ of the interior path is in the images denoted just by $i$ in order to make labels easier to see).

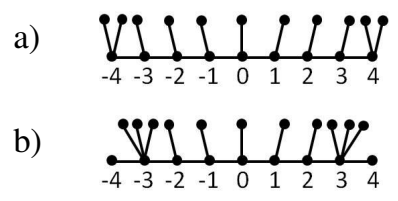

Figure 2: Caterpillar graphs: a) $B_{2}(20,4,1)$, b) $B_{2}(20,4,3)$.

Lemma 2.8. Let $n, d$ and $x$ be integers such that $B_{2}(n, d, x)$ is defined. Then

$$
W\left(B_{2}(n, d, x)\right)=\left(\frac{d}{2}+1\right) n^{2}+(-2 d-2) n-\frac{8 d^{3}}{3}+\frac{32 d}{3}-5+8 x-8 d x-2 x^{2} .
$$

Proof. Let $k=d-1$ and $r=\frac{n-4 d-2 x+2}{2}$. To obtain the exact formula for $W\left(B_{2}(n, d, x)\right)$ we divide vertices from $B_{2}(n, d, x)$ into four sets: set $A$ contains vertices $u_{i}$ for $i=$ 
$-d, \ldots, d$, set $B$ contains leaves appended to $2 k-1$ consecutive vertices $u_{-(k-1)}, \ldots, u_{k-1}$, set $C$ contains $x$ leaves appended to each of the $u_{-(d-1)}$ and $u_{(d-1)}$, while finally set $D$ contains $r$ leaves appended to each of the $u_{-d}$ and $u_{d}$. Note that

$$
\begin{aligned}
& d(A, A)=\sum_{i=-d}^{d} \sum_{j=i+1}^{d}(j-i), d(B, B)=\sum_{i=-(k-1)}^{k-1} \sum_{j=i+1}^{k-1}(j-i+2)+ \\
& d(C, C)=4\left(\begin{array}{l}
x \\
2
\end{array}\right)+x^{2}(2 d), d(D, D)=4\left(\begin{array}{l}
r \\
2
\end{array}\right)+r^{2}(2 d+2)
\end{aligned}
$$

Also, we have

$$
\begin{aligned}
& d(A, B)=\sum_{i=-d}^{d} \sum_{j=-(k-1)}^{k-1}(|i-j|+1), d(A, C)=2 x\left(3+\sum_{i=3}^{2 d+1}(i-1)\right) \\
& d(A, D)=2 r \sum_{i=1}^{2 d+1} i, d(B, C)=2 x \sum_{i=-(k-1)}^{k-1}(i+d+1) \\
& d(B, D)=2 r \sum_{i=-(k-1)}^{k-1}(i+d+2), d(C, D)=2(3 x r+x r(2 d+1)) .
\end{aligned}
$$

Noting that

$$
\begin{aligned}
W\left(B_{1}(n, d, x)\right) & =d(A, A)+d(B, B)+d(C, C)+d(D, D)+ \\
& +d(A, B)+d(A, C)+d(A, D)+ \\
& +d(B, C)+d(B, D)+d(C, D)
\end{aligned}
$$

and simplifying the obtained sum yields the formula from the statement of the lemma. that

Finally, let us denote $d_{1}^{\min }=\left\lceil\frac{n-2}{4}\right\rceil$ and $x_{1}^{\max }=\frac{4+4 d_{1}^{\min }-n}{2}$, while $d_{2}^{\max }=\left\lfloor\frac{n}{4}\right\rfloor$. Note

$$
B_{1}\left(n, d_{1}^{\min }, x_{1}^{\max }\right)=B_{2}\left(n, d_{2}^{\max }, 1\right) .
$$

This equality will provide us with a nice transition from caterpillars based on $B_{1}(n, d, x)$ to caterpillars based on $B_{2}(n, d, x)$ in the following sections.

\section{Even number of vertices}

In this section we will first introduce a special kind of caterpillar based on $B_{1}(n, d, x)$ which will have a longer interior path, then we will introduce a second special kind of caterpillar based on $B_{2}(n, d, x)$ which will have a shorter interior path. For each of those two special kinds of caterpillars we will establish a bound on the value of $d$ for which the interval between values of the Wiener index for two consecutive values of $x$ and $d$ is Wiener 4-complete. The equality (2.1) will then enable us to "glue" all those intervals into one big Wiener 4-complete interval.

Definition 3.1. Let $n, d$ and $x$ be integers for which $B_{1}(n-2, d, x)$ is defined. For $s=$ $-1,0,1,2$ caterpillar $T_{1}(n, d, x, s)$ is a caterpillar on even number of vertices $n$, obtained from $B_{1}(n-2, d, x)$ by appending a leaf to the vertex $u_{s}$ and a leaf to the vertex $u_{d}$ of the path $P=u_{-d} \ldots u_{-1} u_{0} u_{1} \ldots u_{d}$ in $B_{1}(n-2, d, x)$. 
a)

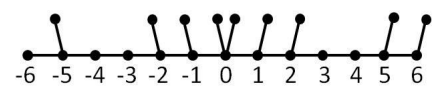

b)

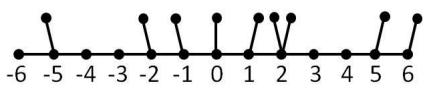

Figure 3: Caterpillar graphs: a) $T_{1}(22,6,2,0)$, b) $T_{1}(22,6,2,2)$.

Caterpillar graph $T_{1}(n, d, x, s)$ is illustrated by Figure 3 (vertex $u_{i}$ of the path $P$ is in the images denoted just by $i$ in order to make labels easier to see).

Lemma 3.2. Let $n, d, x$ and $s$ be integers for which $T_{1}(n, d, x, s)$ is defined. Then

$$
W\left(T_{1}(n, d, x, s)\right)=W\left(B_{1}(n-2, d, x)\right)+\frac{n^{2}}{4}+\frac{3 n}{2}+2 d^{2}+3 d+2 s^{2}-s-2 x .
$$

Proof. Let $k=\frac{(n-2)-(2 d+1)-1}{2}, x^{\prime}=-d-1+x$. We define a function

$$
\begin{gathered}
f(v)=\sum_{i=-d}^{d}(|v-i|+1)+\sum_{i=-(k-1)}^{k-1}(|v-i|+2)+ \\
\left(\left|x^{\prime}-v\right|+2+\left|-x^{\prime}-v\right|+2\right)
\end{gathered}
$$

Now, the definition of $T_{1}(n, d, x, s)$ implies

$$
W\left(T_{1}(n, d, x, s)\right)=W\left(B_{1}(n-2, d, x)\right)+f(s)+f(d)+d-s+2 .
$$

Plugging $s$ and $d$ into the formula for $f$ and simplifying the obtained expression yields the result.

As a direct consequence of Lemma 3.2 we obtain the following corollary.

Corollary 3.3. It holds that

$$
\begin{aligned}
W\left(T_{1}(n, d, x, 1)\right) & =W\left(T_{1}(n, d, x, 0)\right)+1, \\
W\left(T_{1}(n, d, x, 2)\right) & =W\left(T_{1}(n, d, x, 0)\right)+6, \\
W\left(T_{1}(n, d, x,-1)\right) & =W\left(T_{1}(n, d, x, 0)\right)+3 .
\end{aligned}
$$

The main tool in proving the results will be Transformation A of the graph, which, for a given graph, finds another graph whose value of the Wiener index is greater by 4 . Therefore, it is critical to find a graph on which Transformation A can be applied consecutively as many times as possible. That was the basic idea behind constructing graph $T_{1}(n, d, x, s)$ as we did, so that we can use Theorem 2.4 in filling the interval between values $W\left(T_{1}(n, d, x, s)\right)$ for consecutive values of $x$ and $d$. So, let us first apply Theorem 2.4 (i.e. find the corresponding value of $k$ ) to the graph $T_{1}(n, d, x, s)$.

Lemma 3.4. Let $n, d, x$ and $s$ be integers for which $T_{1}(n, d, x, s)$ is defined. For $k=$ $\frac{1}{2} n-d-4$ the interval $\left[W\left(T_{1}(n, d, x, s)\right), W\left(T_{1}(n, d, x, s)\right)+4 k^{2}\right]$ is Wiener 4 -complete. 
Proof. Let us denote $k_{1}=\frac{(n-2)-(2 d+1)-1}{2}$. Note that $k_{1}$ is half of the number of leaves appended to the vertices $u_{ \pm j}$ of the interior path of $T_{1}(n, d, x, s)$ for $j=0, \ldots, k-1$. Since $s \leq 2$, note that the definition of $T_{1}(n, d, x, s)$ and Theorem 2.4 imply the result for $k=k_{1}-2$.

So, let us now establish for which values of $d$ the gap between $W\left(T_{1}(n, d, x, s)\right)$ and $W\left(T_{1}(n, d, x-1, s)\right)$ is smaller than $4 k^{2}$ which is the width of an interval which can be filled by repeatedly applying Transformation $\mathrm{A}$ on $T_{1}(n, d, x, s)$ (i.e. by using Lemma 3.4).

Lemma 3.5. Let $n, d, x \geq 2$ and $s$ be integers for which $T_{1}(n, d, x, s)$ is defined. For $d \leq \frac{1}{2}(n-\sqrt{2 n-8}-8)$ the interval

$$
\left[W\left(T_{1}(n, d, x, s)\right), W\left(T_{1}(n, d, x-1, s)\right)\right]
$$

is Wiener 4-complete.

Proof. First note that

$$
\begin{aligned}
W\left(T_{1}(n, d, x-1, s)\right)-W\left(T_{1}(n, d, x, s)\right) & \leq W\left(T_{1}(n, d, 2, s)\right)-W\left(T_{1}(n, d, 1, s)\right)= \\
& =2(n-5)+2 .
\end{aligned}
$$

Therefore, Lemma 3.4 implies it is sufficient to find integers $n$ and $d$ for which it holds that $4 k^{2} \geq 2(n-5)+2$ where $k=\frac{1}{2} n-d-4$. By a simple calculation it is easy to establish that the inequality holds for $d \leq \frac{1}{2}(n-\sqrt{2 n-8}-8)$ so the lemma is proved.

It is easy to show, using Lemma 3.2, that $W\left(T_{1}(n, d, x-1, s)\right)-W\left(T_{1}(n, d, x, s)\right)=$ $2 n-4 x$ which is divisible by 4 since $n$ is even. Therefore, Lemma 3.5 enables us to "glue" together Wiener 4-complete intervals

$$
\left[W\left(T_{1}(n, d, x, s)\right), W\left(T_{1}(n, d, x-1, s)\right)\right]
$$

into one bigger Wiener 4-complete interval

$$
\left[W\left(T_{1}\left(n, d, x_{1}^{\max }, s\right)\right), W\left(T_{1}(n, d, 1, s)\right)\right]
$$

where $x_{1}^{\max }=\frac{4+4 d-(n-2)}{2}$. Corollary 3.3 then implies that roughly the same interval will be Wiener complete when we take values for every $s=-1,0,1,2$. We say "roughly" because the difference $W\left(T_{1}(n, d, x, 2)\right)=W\left(T_{1}(n, d, x, 0)\right)+6$ makes one point gap at $W\left(T_{1}\left(n, d, x_{1}^{\max }, 0\right)\right)+2$. We now want to "glue" together such bigger intervals into one interval on the border between $d$ and $d-1$. The problem is that

$$
T_{1}\left(n, d, x_{1}^{\max }, s\right) \neq T_{1}(n, d-1,1, s),
$$

so we have to cover the gap in between. Moreover, it holds that

$$
W\left(T_{1}\left(n, d, x_{1}^{\max }, s\right)\right)-W\left(T_{1}(n, d-1,1, s)\right)=n-3
$$

which is not divisible by 4 . Therefore, we have to find enough graphs whose values of the Wiener index will cover the gap of $n-3$ plus the gap of 6 which arises from the "rough" edge of the interval for a given $d$. 
Lemma 3.6. Let $n, d, x_{1}^{\max }=\frac{4+4 d-(n-2)}{2}$ and $s$ be integers for which $T_{1}\left(n, d, x_{1}^{\max }, s\right)$ and $T_{1}(n, d-1,1, s)$ are defined. For $d \leq \frac{1}{2}(n-\sqrt{n+3}-6)$ the interval

$$
\left[W\left(T_{1}(n, d-1,1, s)\right), W\left(T_{1}\left(n, d, x_{1}^{\max }, s\right)\right)+6\right]
$$

is Wiener 4-complete.

Proof. Since

$$
W\left(T_{1}\left(n, d, x_{1}^{\max }, s\right)\right)+6-W\left(T_{1}(n, d-1,1, s)\right)=n-3+6=n+3,
$$

Lemma 3.4 implies that it is sufficient to find for which $d$ it holds that $4 k^{2} \geq n+3$ where $k=\frac{1}{2} n-(d-1)-4$. By a simple calculation one obtains that inequality holds for $d \leq \frac{1}{2}(n-\sqrt{n+3}-6)$ which proves the theorem.

Note that the restriction on the maximum value of $d$ is stricter in Lemma 3.5 then in Lemma 3.6 for every $n>4$.

Now we have taken out all we could from graph $T_{1}$, but that covers only caterpillars with relatively large $d$. We can further expand the Wiener complete interval to the left side, i.e. to caterpillars with smaller $d$, using graph $T_{2}$ which we will construct from the basic graph $B_{2}$.

Definition 3.7. Let $n, d$ and $x$ be integers for which $B_{2}(n-2, d, x)$ is defined. For $s=$ $-1,0,1,2$ caterpillar $T_{2}(n, d, x, s)$ is a caterpillar on even number of vertices $n$, obtained from $B_{2}(n-2, d, x)$ by appending a leaf to the vertex $u_{s}$ and a leaf to the vertex $u_{d}$ of the path $P=u_{-d} \ldots u_{-1} u_{0} u_{1} \ldots u_{d}$ in $B_{2}(n-2, d, x)$.

Caterpillar graph $T_{2}(n, d, x, s)$ is illustrated by Figure 4 (vertex $u_{i}$ of the path $P$ is in the images denoted just by $i$ in order to make labels easier to see).

a)

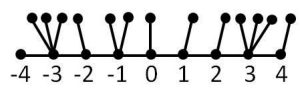

b)

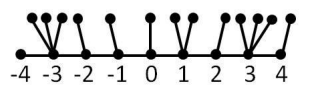

Figure 4: Caterpillar graphs: a) $T_{2}(22,4,3,-1)$, b) $T_{2}(22,4,3,1)$.

Lemma 3.8. Let $n, d, x$ and $s$ be integers for which $T_{2}(n, d, x, s)$ is defined. Then

$$
W\left(T_{2}(n, d, x, s)\right)=W\left(B_{2}(n-2, d, x)\right)+(2 d+4) n-2 d^{2}-7 d-6-2 x+2 s^{2}-s .
$$

Proof. Let $k=d-1$ and $r=\frac{n-4 d-2 x}{2}$. We define a function

$$
\begin{aligned}
f(v) & =\sum_{i=-d}^{d}(|v-i|+1)+\sum_{i=-(k-1)}^{k-1}(|v-i|+2)+ \\
& +x(|v+(d-1)|+2)+x(|v-(d-1)|+2)+ \\
& +r(|v+d|+2)+r(|v-d|+2) .
\end{aligned}
$$


Now, the definition of $T_{2}(n, d, x, s)$ implies

$$
W\left(T_{2}(n, d, x, s)\right)=W\left(B_{2}(n-2, d, x)\right)+f(s)+f(d)+d-s+2 .
$$

Plugging $s$ and $d$ into the formula for $f$ and simplifying the obtained expression yields the result.

Again, as a direct consequence of Lemma 3.8 we obtain the following corollary.

Corollary 3.9. It holds that

$$
\begin{aligned}
W\left(T_{2}(n, d, x, 1)\right) & =W\left(T_{2}(n, d, x, 0)\right)+1, \\
W\left(T_{2}(n, d, x, 2)\right) & =W\left(T_{2}(n, d, x, 0)\right)+6, \\
W\left(T_{2}(n, d, x,-1)\right) & =W\left(T_{2}(n, d, x, 0)\right)+3 .
\end{aligned}
$$

As in the case of large $d$, the main tool in obtaining the results will be the following lemma.

Lemma 3.10. Let $n, d, x$ and $s$ be integers for which $T_{2}(n, d, x, s)$ is defined. For $k=d-3$ the interval $\left[W\left(T_{2}(n, d, x, s)\right), W\left(T_{2}(n, d, x, s)\right)+4 k^{2}\right]$ is Wiener 4-complete.

Proof. Let us denote $k_{1}=d-1$. Note that $k_{1}$ is half of the number of leaves appended to the vertices $u_{ \pm j}$ of the interior path of $T_{2}(n, d, x, s)$ for $j=0, \ldots, k-1$. Since $s \leq 2$, note that the definition of $T_{2}(n, d, x, s)$ and Theorem 2.4 imply the result for $k=k_{1}-2$.

We will first use Lemma 3.10 to cover the interval between $W\left(T_{2}(n, d, x, s)\right)$ and $W\left(T_{2}(n, d, x-1, s)\right)$, after that we will use it to cover the gap between $W\left(T_{2}(n, d-\right.$ $1,1, s))$ and $W\left(T_{2}\left(n, d, x_{2}^{\max }, s\right)\right)$.

Lemma 3.11. Let $n, d, x \geq 2$ and $s$ be integers for which $T_{2}(n, d, x, s)$ is defined. For $d \geq \frac{1}{2}(\sqrt{2 n-8}+6)$ the interval

$$
\left[W\left(T_{2}(n, d, x, s)\right), W\left(T_{2}(n, d, x-1, s)\right)\right]
$$

is Wiener 4-complete.

Proof. First note that for $x_{2}^{\max }=\frac{(n-2)-4 d+2}{2}$ it holds that

$$
\begin{aligned}
& W\left(T_{2}(n, d, x-1, s)\right)-W\left(T_{2}(n, d, x, s)\right) \leq \\
& \leq W\left(T_{2}\left(n, d, x_{2}^{\max }-1, s\right)\right)-W\left(T_{2}\left(n, d, x_{2}^{\max }, s\right)\right)= \\
& =2(n-5)+2 .
\end{aligned}
$$

Therefore, Lemma 3.10 implies it is sufficient to find for which $n$ and $d$ it holds that $4 k^{2} \geq$ $2(n-5)+2$ where $k=d-3$. By a simple calculation it is easy to establish that the inequality holds for $d \geq \frac{1}{2}(\sqrt{2 n-8}+6)$ so the theorem is proved.

Again, it is easy to show that $W\left(T_{2}(n, d, x-1, s)\right)-W\left(T_{2}(n, d, x, s)\right)=4 d+4 x-8$ which is divisible by 4 . Therefore, using Lemma 3.11 we can again "glue" the interval for different values of $x$ into one bigger interval which will be "roughly" Wiener complete when taking values of $W\left(T_{2}(n, d, x, s)\right)$ for every $s=-1,0,1,2$. The next thing is to cover the gap between $W\left(T_{2}(n, d-1,1, s)\right)$ and $W\left(T_{2}\left(n, d, x_{2}^{\max }, s\right)\right)$ which equals $n-3$ plus the gap of 6 which arises from the "rough" ends of the Wiener complete interval for given $n$ and $d$. 
Lemma 3.12. Let $n, d, x_{2}^{\max }=\frac{(n-2)-4 d+2}{2}$ and $s$ be integers for which $T_{2}\left(n, d, x_{2}^{\max }, s\right)$ and $T_{2}(n, d-1,1, s)$ is defined. For $d \geq \frac{1}{2}(\sqrt{n+3}+8)$ the interval

$$
\left[W\left(T_{2}(n, d-1,1, s)\right), W\left(T_{2}\left(n, d, x_{2}^{\max }, s\right)\right)+6\right]
$$

is Wiener 4-complete.

Proof. Since

$$
W\left(T_{2}\left(n, d, x_{2}^{\max }, s\right)\right)+6-W\left(T_{2}(n, d-1,1, s)\right)=n+3,
$$

Lemma 3.10 implies it is sufficient to find $n$ and $d$ for which it holds that $4 k^{2} \geq n+3$ where $k=(d-1)-3$. By a simple calculation one obtains that the inequality holds for $d \geq \frac{1}{2}(\sqrt{n+3}+8)$ which proves the theorem.

Therefore, using graphs $T_{1}(n, d, x, s)$ and $T_{2}(n, d, x, s)$ we obtained two big Wiener complete intervals, which it would be nice if we could "glue" together into one big Wiener complete interval. In order to do that, note that the equality (2.1) implies

$$
T_{2}\left(n, d_{2}^{\max }, 1, s\right)=T_{1}\left(n, d_{1}^{\min }, x_{1}^{\max }, s\right)
$$

for $d_{2}^{\max }=\left\lfloor\frac{n-2}{4}\right\rfloor, d_{1}^{\min }=\left\lceil\frac{n-4}{4}\right\rceil$ and $x_{1}^{\max }=\frac{4+4 d_{1}^{\min }-(n-2)}{2}$. Now we can state the theorem which gives us the largest Wiener complete interval we have managed to obtain.

Theorem 3.13. Let $n \geq 30, d_{2}^{\min }=\left\lceil\frac{1}{2}(\sqrt{2 n-8}+6)\right\rceil, x_{2}^{\max }=\frac{(n-2)-4 d_{2}^{\min }+2}{2}$ and $d_{1}^{\max }=\left\lfloor\frac{1}{2}(n-\sqrt{2 n-8}-8)\right\rfloor$. The interval

$$
\left[W\left(T_{2}\left(n, d_{2}^{\min }, x_{2}^{\max }, 2\right)\right), W\left(T_{1}\left(n, d_{1}^{\max }, 1,0\right)\right)\right]
$$

is Wiener complete.

Now that we have obtained very large Wiener complete interval, we can finally prove the following theorem which is our main result and which proves Conjectures 1.1 and 1.2 in terms of $n^{3}$.

Theorem 3.14. For even $n \geq 30$ it holds that $\left|W^{i n t}\left[\mathcal{T}_{n}\right]\right|=\left|W\left[\mathcal{T}_{n}\right]\right|=\frac{1}{6} n^{3}+O\left(n^{5 / 2}\right)$.

Proof. Theorem 3.13 implies

$$
\left|W\left[\mathcal{T}_{n}\right]\right| \geq\left|W^{\text {int }}\left[\mathcal{T}_{n}\right]\right| \geq W\left(T_{1}\left(n, d_{1}^{\max }, 1,0\right)\right)-W\left(T_{2}\left(n, d_{2}^{\min }, x_{2}^{\max }, 2\right)\right)
$$

where $d_{2}^{\min }=\frac{1}{2}(\sqrt{2 n-8}+6)+p, x_{2}^{\max }=\frac{(n-2)-4 d_{2}^{\min }+2}{2}$ and $d_{1}^{\max }=\frac{1}{2}(n-\sqrt{2 n-8}-$ $8)-1+q$ for $p \in[0,1\rangle$ and $q \in\langle 0,1]$. From Lemmas 3.2 and 3.8 we further obtain that

$$
\left|W^{i n t}\left[\mathcal{T}_{n}\right]\right| \geq \frac{1}{6} n^{3}-\frac{1}{2} \sqrt{2 n^{5}-8 n^{4}}-4 n^{2}+\frac{10}{3} \sqrt{2 n^{3}-8 n^{2}}+\frac{143}{6} n+21 \sqrt{2 n-8}-51 .
$$

On the other hand, recall that $\left|W^{i n t}\left[\mathcal{T}_{n}\right]\right| \leq\left|W\left[\mathcal{T}_{n}\right]\right| \leq W\left(P_{n}\right)-W\left(S_{n}\right)+1=\frac{1}{6} n^{3}-$ $n^{2}+\frac{11}{6} n$, which proves the theorem. 
Note that caterpillar trees $T_{1}(n, d, x, s)$ are chemical trees (i.e. trees in which the degree of every vertex is at most 4 ) for all possible values of its parameters and they remain chemical after repeated application of Transformation A. Therefore, half of these results hold for chemical trees and we obtain the following corollary.

Corollary 3.15. Let $\mathcal{T}_{n}^{4}$ be a class of chemical trees on $n$ vertices where $n \geq 30$ is even. Then $\left|W^{\text {int }}\left[\mathcal{T}_{n}^{4}\right]\right|=\left|W\left[\mathcal{T}_{n}^{4}\right]\right|=\Theta\left(n^{3}\right)$.

Proof. Note that Lemmas 3.5 and 3.6 imply that

$$
\left|W\left[\mathcal{T}_{n}^{4}\right]\right| \geq\left|W^{i n t}\left[\mathcal{T}_{n}^{4}\right]\right| \geq W\left(T_{1}\left(n, d_{1}^{\max }, 1,0\right)\right)-W\left(T_{1}\left(n, d_{1}^{\min }, x_{1}^{\max }, 2\right)\right)
$$

where $d_{1}^{\max }=\frac{1}{2}(n-\sqrt{2 n-8}-8)-1+p, d_{1}^{\min }=\frac{n-2}{4}+q$ and $x_{1}^{\max }=\frac{6+4 d_{1}^{\min }-n}{2}$ for $p \in\langle 0,1]$ and $q \in[0,1\rangle$. From Lemma 3.2 we obtain

$$
\left|W^{i n t}\left[\mathcal{T}_{n}^{4}\right]\right| \geq \frac{1}{12} n^{3}-\frac{1}{4} \sqrt{2 n^{5}-8 n^{4}}-\frac{15}{8} n^{2}+\frac{5}{3} \sqrt{2 n^{3}-8 n^{2}}+\frac{101}{12} n+\frac{9}{2} \sqrt{2 n-8}-\frac{134}{3} .
$$

Note that this result for chemical trees is obtained using only chemical trees with relatively large diameter and the result is still the best possible with regard to the highest power $n^{3}$ (just the power, not the coefficient). This means that this result is something that probably can be significantly improved by considering chemical trees with shorter diameter, but we leave that as an open problem for future research.

\section{Odd number of vertices}

The strategy to prove the result in the case of odd number of vertices is the same as in the case of even number of vertices. The only difference is that in this case the value of the Wiener index can be only even number so we are aiming at the largest possible interval of consecutive even numbers which are values of the Wiener index for a tree.

Definition 4.1. Let $n, d$ and $x$ be integers for which $B_{1}(n-1, d, x)$ is defined. For $s=$ 0,1 caterpillar $T_{3}(n, d, x, s)$ is a caterpillar on odd number of vertices $n$, obtained from $B_{1}(n-1, d, x)$ by appending a leaf to the vertex $u_{s}$ of the path $P=u_{-d} \ldots u_{-1} u_{0} u_{1} \ldots u_{d}$ in $B_{1}(n-1, d, x)$.

Caterpillar tree $T_{3}(n, d, x, s)$ is illustrated by Figure 5 (vertex $u_{i}$ of the path $P$ is in the images denoted just by $i$ in order to make labels easier to see).

a)

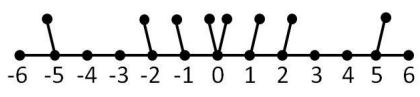

b)

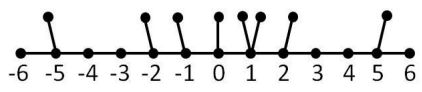

Figure 5: Caterpillar graphs: a) $T_{3}(21,6,2,0)$, b) $T_{3}(21,6,2,1)$. 
Lemma 4.2. Let $n, d, x$ and $s$ be integers for which $T_{3}(n, d, x, s)$ is defined. Then

$$
W\left(T_{3}(n, d, x, s)\right)=W\left(B_{1}(n-1, d, x)\right)+\frac{n^{2}}{4}-d n+2 d^{2}+5 d+\frac{11}{4}-2 x+2 s^{2} .
$$

Proof. Let $k=\frac{(n-1)-(2 d+1)-1}{2}, x^{\prime}=-d-1+x$. The definition of $T_{3}(n, d, x, s)$ implies

$$
\begin{aligned}
W\left(T_{3}(n, d, x, s)\right)= & W\left(B_{1}(n-1, d, x)\right)+\sum_{i=-d}^{d}(|s-i|+1)+ \\
& +\sum_{i=-(k-1)}^{k-1}(|s-i|+2)+\left(s-x^{\prime}+2\right)+ \\
& +\left(-x^{\prime}-s+2\right) .
\end{aligned}
$$

Simplifying this expression yields the result.

As a direct consequence of Lemma 4.2 we obtain the following corollary.

Corollary 4.3. It holds that $W\left(T_{3}(n, d, x, 1)\right)=W\left(T_{3}(n, d, x, 0)\right)+2$.

We now want to apply Theorem 2.4 to $T_{3}(n, d, x, s)$, i.e. we want to establish the value of $k$ in the case of this special graph.

Lemma 4.4. Let $n, d, x$ and $s$ be integers for which $T_{3}(n, d, x, s)$ is defined. For $k=$ $\frac{1}{2} n-d-\frac{5}{2}$ the interval $\left[W\left(T_{3}(n, d, x, s)\right), W\left(T_{3}(n, d, x, s)\right)+4 k^{2}\right]$ is Wiener 4-complete.

Proof. Let us denote $k_{1}=\frac{(n-1)-(2 d+1)-1}{2}$. Note that $k_{1}$ is half of the number of leaves appended to the vertices $u_{ \pm j}$ of the interior path of $T_{3}(n, d, x, s)$ for $j=0, \ldots, k-1$. Since $s \leq 1$, note that the definition of $T_{3}(n, d, x, s)$ and Theorem 2.4 imply the result for $k=k_{1}-1$.

So, let us now establish for which values of $d$ the gap between $W\left(T_{3}(n, d, x, s)\right)$ and $W\left(T_{3}(n, d, x-1, s)\right)$ is smaller than $4 k^{2}$ where $k=\frac{1}{2} n-d-\frac{5}{2}$.

Lemma 4.5. Let $n, d, x \geq 2$ and $s$ be integers for which $T_{3}(n, d, x, s)$ is defined. For $d \leq \frac{1}{2}(n-\sqrt{2 n-6}-5)$ the interval

$$
\left[W\left(T_{3}(n, d, x, s)\right), W\left(T_{3}(n, d, x-1, s)\right)\right]
$$

is Wiener 4-complete.

Proof. First note that

$$
\begin{aligned}
T_{3}(n, d, x-1, s)-T_{3}(n, d, x, s) & \leq T_{3}(n, d, 2, s)-T_{3}(n, d, 1, s)= \\
& =2(n-4)+2 .
\end{aligned}
$$

Therefore, Lemma 4.4 implies it is sufficient to find integers $n$ and $d$ for which it holds that $4 k^{2} \geq 2(n-4)+2$ where $k=\frac{1}{2} n-d-\frac{5}{2}$. By a simple calculation it is easy to establish that the inequality holds for $d \leq \frac{1}{2}(n-\sqrt{2 n-6}-5)$ so the lemma is proved. 
Using Lemma 4.2 it is easy to establish that

$$
W\left(T_{3}(n, d, x-1, s)\right)-W\left(T_{3}(n, d, x, s)\right)=2(n-2 x+1)
$$

which is divisible by 4 since $n$ is odd. Moreover, note that for $x_{3}^{\max }=\frac{4+4 d-(n-1)}{2}$ it holds that

$$
T_{3}\left(n, d, x_{3}^{\max }, s\right)=T_{3}(n, d-1,1, s) .
$$

Therefore we can use Lemma 4.5 and "glue" together intervals both on the border between $x$ and $x-1$ and on the border of $d$ and $d-1$, so we will obtain one large interval which is Wiener 2-complete (because of Corollary 4.3).

Again, here we have used $T_{3}(n, d, x, s)$ to the maximum, but we have covered thus only caterpillars with large $d$. Let us now use graph $B_{2}(n, d, x)$ to create the fourth special kind of caterpillars which we will use to widen our interval to caterpillars with small $d$.

Definition 4.6. Let $n, d$ and $x$ be integers for which $B_{2}(n-1, d, x)$ is defined. For $s=$ 0,1 caterpillar $T_{4}(n, d, x, s)$ is a caterpillar on odd number of vertices $n$, obtained from $B_{2}(n-1, d, x)$ by appending a leaf to the vertex $u_{s}$ of the path $P=u_{-d} \ldots u_{-1} u_{0} u_{1} \ldots u_{d}$ in $B_{2}(n-1, d, x)$.

Caterpillar graph $T_{3}(n, d, x, s)$ is illustrated by Figure 6 (vertex $u_{i}$ of the path $P$ is in the images denoted just by $i$ in order to make labels easier to see).

a)

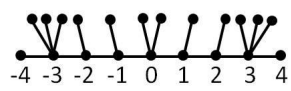

b)

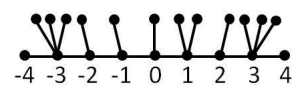

Figure 6: Caterpillar graphs: a) $T_{4}(21,4,3,0)$, b) $T_{4}(21,4,3,1)$.

Lemma 4.7. Let $n, d, x$ and $s$ be integers for which $T_{4}(n, d, x, s)$ is defined. Then

$$
W\left(T_{4}(n, d, x, s)\right)=W\left(B_{2}(n-1, d, x)\right)+(2+d) n-2 d^{2}-3 d-1+2 s^{2}-2 x .
$$

Proof. Let $k=d-1$ and $r=\frac{(n-1)-4 d-2 x+2}{2}$. The definition of $T_{4}(n, d, x, s)$ implies

$$
\begin{aligned}
W\left(T_{4}(n, d, x, s)\right)= & W\left(B_{2}(n-1, d, x)\right)+\sum_{i=-d}^{d}(|s-i|+1)+ \\
& +\sum_{i=-(k-1)}^{k-1}(|s-i|+2)+\left(s-x^{\prime}+2\right)+ \\
& +2 x(d+1)+2 r(d+2) .
\end{aligned}
$$

Simplifying this expression yields the result.

Corollary 4.8. It holds that $W\left(T_{4}(n, d, x, 1)\right)=W\left(T_{4}(n, d, x, 0)\right)+2$. 
Let us now apply Theorem 2.4 to $T_{4}(n, d, x, s)$.

Lemma 4.9. Let $n, d, x$ and $s$ be integers for which $T_{4}(n, d, x, s)$ is defined. For $k=d-2$ the interval $\left[W\left(T_{4}(n, d, x, s)\right), W\left(T_{4}(n, d, x, s)\right)+4 k^{2}\right]$ is Wiener 4-complete.

Proof. Let us denote $k_{1}=d-1$. Note that $k_{1}$ is half of the number of leaves appended to the vertices $u_{ \pm j}$ of the interior path of $T_{3}(n, d, x, s)$ for $j=0, \ldots, k-1$. Since $s \leq 1$, note that the definition of $T_{4}(n, d, x, s)$ and Theorem 2.4 imply the result for $k=k_{1}-1$.

Now we can establish the minimum value of $d$ for which the difference between Wiener index of $T_{4}(n, d, x, s)$ and $T_{4}(n, d, x-1, s)$ can be "covered" by Transformation A.

Lemma 4.10. Let $n, d, x \geq 2$ and $s$ be integers for which $T_{4}(n, d, x, s)$ is defined. For $d \geq \frac{1}{2}(\sqrt{2 n-6}+4)$ the interval

$$
\left[W\left(T_{4}(n, d, x, s)\right), W\left(T_{4}(n, d, x-1, s)\right)\right]
$$

is Wiener 4-complete.

Proof. First note that for $x_{4}^{\max }=\frac{(n-1)-4 d+2}{2}$ it holds that

$$
\begin{aligned}
& W\left(T_{4}(n, d, x-1, s)\right)-W\left(T_{4}(n, d, x, s)\right) \\
& \leq W\left(T_{4}\left(n, d, x_{4}^{\max }-1, s\right)\right)-W\left(T_{4}\left(n, d, x_{4}^{\max }, s\right)\right)= \\
& =2(n-4)+2 .
\end{aligned}
$$

Therefore, Lemma 4.9 implies it is sufficient to find integers $n$ and $d$ for which it holds that $4 k^{2} \geq 2(n-4)+2$ where $k=d-2$. By a simple calculation it is easy to establish that the inequality holds for $d \geq \frac{1}{2}(\sqrt{2 n-6}+4)$ so the lemma is proved.

Using Lemma 4.7 it is easy to establish that

$$
W\left(T_{4}(n, d, x-1, s)\right)-W\left(T_{4}(n, d, x, s)\right)=4(x+2 d-2)
$$

which is divisible by 4 . Moreover, note that for $x_{4}^{\max }=\frac{(n-1)-4 d+2}{2}$ it holds that

$$
T_{4}\left(n, d, x_{4}^{\max }, s\right)=T_{4}(n, d-1,1, s) .
$$

Therefore we can use Lemma 4.10 and "glue" together intervals both on the border between $x$ and $x-1$ and on the border of $d$ and $d-1$, so we will obtain one large interval which is Wiener 2-complete (because of Corollary 4.8).

Finally, noting that for $d_{3}^{\min }=\left\lceil\frac{n-3}{4}\right\rceil, x_{3}^{\max }=\frac{4+4 d_{3}^{\min }-(n-1)}{2}$ and $d_{4}^{\max }=\left\lfloor\frac{n-1}{4}\right\rfloor$ it holds that

$$
T_{3}\left(n, d_{3}^{\min }, x_{3}^{\max }, s\right)=T_{4}\left(n, d_{4}^{\max }, 1, s\right),
$$

we conclude that we can "glue" together two large Wiener 2-complete intervals we obtained (one for large values of $d$ and the other for small values of $d$ ), and thus we obtain the following theorem which gives us the largest Wiener 2-complete interval we manage to obtain. 
Theorem 4.11. Let $n \geq 21, d_{3}^{\max }=\left\lfloor\frac{1}{2}(n-\sqrt{2 n-6}-5)\right\rfloor, d_{4}^{\min }=\left\lceil\frac{1}{2}(\sqrt{2 n-6}+4)\right\rceil$ and $x_{4}^{\max }=\frac{(n-1)-4 d_{4}^{\min }+2}{2}$. The interval

$$
\left[W\left(T_{4}\left(n, d_{4}^{\min }, x_{4}^{\max }, 1\right)\right), W\left(T_{3}\left(n, d_{3}^{\max }, 1,0\right)\right)\right]
$$

is Wiener 2-complete.

Having the largest Wiener 2-complete interval from the previous theorem, we can now finally prove the following theorem which is our main result and which proves Conjectures 1.1 and 1.2 in terms of $n^{3}$.

Theorem 4.12. For odd $n \geq 21$ it holds that $\left|W^{\text {int }}\left[\mathcal{T}_{n}\right]\right|=\left|W\left[\mathcal{T}_{n}\right]\right|=\frac{1}{12} n^{3}+O\left(n^{5 / 2}\right)$.

Proof. Using Theorem 3.13 we obtain

$$
\left|W\left[\mathcal{T}_{n}\right]\right| \geq\left|W^{\text {int }}\left[\mathcal{T}_{n}\right]\right| \geq\left(W\left(T_{3}\left(n, d_{3}^{\max }, 1,0\right)\right)-W\left(T_{4}\left(n, d_{4}^{\min }, x_{4}^{\max }, 1\right)\right)\right) / 2
$$

where $d_{3}^{\max }=\frac{1}{2}(n-\sqrt{2 n-6}-5)-1+p, d_{4}^{\min }=\frac{1}{2}(\sqrt{2 n-6}+4)+q$ and $x_{4}^{\max }=$ $\frac{(n-1)-4 d_{4}^{\min }+2}{2}$ for $p \in\langle 0,1]$ and $q \in[0,1\rangle$. Now, using Lemmas 4.2 and 4.7 we further obtain

$$
\begin{aligned}
\left|W\left[\mathcal{T}_{n}\right]\right| \geq & \left|W^{i n t}\left[\mathcal{T}_{n}\right]\right| \geq \frac{1}{12} n^{3}-\frac{1}{4} \sqrt{2 n^{5}-6 n^{4}}-\frac{3}{2} n^{2}+\frac{5}{3} \sqrt{2 n^{3}-6 n^{2}}+ \\
& +\frac{83}{12} n-\frac{13}{12} \sqrt{2 n-6}-\frac{253}{12} .
\end{aligned}
$$

On the other hand, Theorem 2.1 implies $\left|W^{\text {int }}\left[\mathcal{T}_{n}\right]\right| \leq\left|W\left[\mathcal{T}_{n}\right]\right| \leq\left(W\left(P_{n}\right)-W\left(S_{n}\right)+\right.$ 1) $/ 2=\frac{1}{12} n^{3}-\frac{1}{2} n^{2}+\frac{11}{12} n$.

Again, since caterpillars $T_{3}(n, d, x, s)$ are chemical trees for all possible values of parameters, and remain chemical after applying repeatedly Transformation A, half of these results hold for chemical trees, i.e. we obtain the following corollary.

Corollary 4.13. Let $\mathcal{T}_{n}^{4}$ be a class of chemical trees on $n$ vertices where $n \geq 21$ is odd. Then $\left|W^{\text {int }}\left[\mathcal{T}_{n}^{4}\right]\right|=\left|W\left[\mathcal{T}_{n}^{4}\right]\right|=\Theta\left(n^{3}\right)$.

Proof. Using Lemma 4.5 we obtain

$$
\left|W\left[\mathcal{T}_{n}^{4}\right]\right| \geq\left|W^{i n t}\left[\mathcal{T}_{n}^{4}\right]\right| \geq\left(W\left(T_{3}\left(n, d_{3}^{\max }, 1,0\right)\right)-W\left(T_{3}\left(n, d_{3}^{\min }, x_{3}^{\max }, 1\right)\right)\right) / 2
$$

where $d_{3}^{\min }=\frac{n-3}{4}+p, x_{3}^{\max }=\frac{4+4 d_{3}^{\min }-(n-1)}{2}$ and $d_{3}^{\max }=\frac{1}{2}(n-\sqrt{2 n-6}-5)-1+q$ for $p \in[0,1\rangle$ and $q \in\langle 0,1]$. Using Lemma 4.2 we further obtain

$$
\left|W^{i n t}\left[\mathcal{T}_{n}^{4}\right]\right| \geq \frac{1}{24} n^{3}-\frac{1}{8} \sqrt{2 n^{5}-6 n^{4}}-\frac{11}{16} n^{2}+\frac{5}{6} \sqrt{2 n^{3}-6 n^{2}}+\frac{19}{12} n-\frac{61}{24} \sqrt{2 n-6}-\frac{685}{48} .
$$

Again, this result for chemical trees is obtained by considering only chemical trees with large diameter so it probably can be significantly improved, but we leave that as an open problem for future research. 


\section{Conclusion}

In this paper we have proved that in the case of even $n$ the cardinality of the largest interval $W^{\text {int }}\left[\mathcal{T}_{n}\right]$ of consecutive integers which are values of the Wiener index for a tree graph on $n$ vertices equals $\frac{1}{6} n^{3}+O\left(n^{5 / 2}\right)$. In the case of odd $n$ the value of the Wiener index for a tree on $n$ vertices can be only even number, therefore the cardinality of the largest interval $W^{i n t}\left[\mathcal{T}_{n}\right]$ of consecutive even integers which are values of the Wiener index for a tree graph on $n$ vertices equals $\frac{1}{12} n^{3}+O\left(n^{5 / 2}\right)$. Since the set $W^{i n t}\left[\mathcal{T}_{n}\right]$ is a subset of the set $W\left[\mathcal{T}_{n}\right]$ of all values of the Wiener index for trees on $n$ vertices, this immediately yields the same result on the cardinality of $W\left[\mathcal{T}_{n}\right]$. The upper bound $\left|W\left[\mathcal{T}_{n}\right]\right| \leq \frac{1}{6} n^{3}-n^{2}+\frac{11}{6} n$ (i.e. $\left|W\left[\mathcal{T}_{n}\right]\right| \leq \frac{1}{12} n^{3}-\frac{1}{2} n^{2}+\frac{11}{12} n$ for odd $n$ ) is easily established by calculating the difference of the value of the Wiener index for maximal and minimal tree graphs (the path $P_{n}$ and the star $S_{n}$ respectively). Comparing this bound with our results it is readily seen that our results are best possible with respect to $n^{3}$. Yet, with respect to $n^{2}$ the results are not so good, because we obtained $\left|W\left[\mathcal{T}_{n}\right]\right|=\left|W^{\text {int }}\left[\mathcal{T}_{n}\right]\right|=\frac{1}{6} n^{3}+O\left(n^{5 / 2}\right)$ (i.e. $\frac{1}{12} n^{3}+O\left(n^{5 / 2}\right)$ in the case of odd $n$ ). This may be due to the fact that in the paper we aimed at the bound for $\left|W^{\text {int }}\left[\mathcal{T}_{n}\right]\right|$ and we stopped with our search when the interval was interrupted (when the diameter of a tree became too small or too large). There is the possibility that the same approach extended to the caterpillars of all diameters would yield sufficient improvement on $\left|W\left[\mathcal{T}_{n}\right]\right|$ to reduce $O\left(n^{5 / 2}\right)$ to $O\left(n^{2}\right)$. But we leave that for future research.

Furthermore, in our research we focused only on caterpillar trees, so the obvious corollary is that the same results hold in the narrower class of caterpillar trees. Half of the caterpillars we used are chemical trees, which yields relatively strong result for the class of chemical trees as a direct corollary. Also, we researched the caterpillars grouped by the length of the interior path (which is nearly the diameter), so the results for trees with given diameter would also follow easily though it is questionable how strong those results would be. Researching the same question in the classes of trees with other given parameters might also be interesting direction of future research.

\section{References}

[1] Y.-E. A. Ban, S. Bereg and N. H. Mustafa, A conjecture on Wiener indices in combinatorial chemistry, Algorithmica 40 (2004), 99-117, doi:10.1007/s00453-004-1097-y.

[2] A. A. Dobrynin, R. Entringer and I. Gutman, Wiener index of trees: theory and applications, Acta Appl. Math. 66 (2001), 211-249, doi:10.1023/a:1010767517079.

[3] I. Gutman, Frequency of even and odd numbers in distance matrixes of bipartite graphs, $J$. Chem. Inf. Comput. Sci. 34 (1994), 912-914, doi:10.1021/ci00020a027.

[4] I. Gutman and Y.-N. Yeh, The sum of all distances in bipartite graphs, Math. Slovaca 45 (1995), 327-334, http://maslo.mat.savba.sk/paper.php?id_paper=143.

[5] M. Knor, R. Škrekovski and A. Tepeh, Mathematical aspects of Wiener index, Ars Math. Contemp. 11 (2016), 327-352, http: //amc-journal. eu/index.php/amc/article/ view/795.

[6] M. Krnc and R. Škrekovski, On Wiener inverse interval problem, MATCH Commun. Math. Comput. Chem. 75 (2016), 71-80, http://match.pmf.kg.ac.rs/electronic_ versions/Match75/n1/match75n1_71-80.pdf.

[7] M. Lepović and I. Gutman, A collective property of trees and chemical trees, J. Chem. Inf. Comput. Sci. 38 (1998), 823-826, doi:10.1021/ci980004b. 
[8] I. Lukovits, Frequency of even and odd numbers in distance matrices of trees, J. Chem. Inf. Comput. Sci. 33 (1993), 626-629, doi:10.1021/ci00014a016.

[9] S. G. Wagner, A class of trees and its Wiener index, Acta Appl. Math. 91 (2006), 119-132, doi:10.1007/s10440-006-9026-5.

[10] S. G. Wagner and H. Wang, On the parity of the Wiener index, European J. Combin. 30 (2009), 996-1004, doi:10.1016/j.ejc.2008.06.004.

[11] S. G. Wagner, H. Wang and G. Yu, Molecular graphs and the inverse Wiener index problem, Discrete Appl. Math. 157 (2009), 1544-1554, doi:10.1016/j.dam.2008.06.008.

[12] H. Wang and G. Yu, All but 49 numbers are Wiener indices of trees, Acta Appl. Math. 92 (2006), 15-20, doi:10.1007/s10440-006-9037-2.

[13] H. Wiener, Structural determination of paraffin boiling points, J. Am. Chem. Soc. 69 (1947), 17-20, doi:10.1021/ja01193a005.

[14] K. Xu, M. Liu, K. Ch. Das, I. Gutman and B. Furtula, A survey on graphs extremal with respect to distance-based topological indices, MATCH Commun. Math. Comput. Chem. 71 (2014), 461-508, http://match.pmf.kg.ac.rs/electronic_ versions/Match71/n3/match71n3_461-508.pdf. 